\title{
Propiedades Mecánicas de un Material de Pectina para Revestimiento de Fibras Naturales Utilizadas en Aplicaciones Agrícolas
}

\author{
David F. López ${ }^{(1)}$, Oswaldo Osorio(1)* y Oscar E. Checa(2) \\ (1) Universidad de Nariño, Sede Torobajo, Facultad de ingeniería agroindustrial, Grupo de apoyo a la investigación y \\ desarrollo agroalimentario GAIDA, Pasto - Colombia. (e-mail: Osorio_oswaldo@udenar.edu.co) \\ (2) Universidad de Nariño, Sede Torobajo, Facultad de ingeniería agronómica, Grupo de investigación en cultivos \\ andinos, Pasto - Colombia. (e-mail: gricandudenar@gmail.com)
}

Recibido Ago. 20, 2018; Aceptado Nov. 6, 2018; Versión final Dic. 18, 2018, Publicado Jun. 2019

\section{Resumen}

El objetivo de este estudio fue evaluar las propiedades mecánicas porcentaje de elongación (\%) y resistencia a la tracción (MPa) de películas de pectina $(4 \%$ y $6 \% \mathrm{p} / \mathrm{v})$, aceite mineral $(2 \%$ y $6 \% \mathrm{p} / \mathrm{v})$ y glicerol $(3 \%$ y $6 \%$ $\mathrm{p} / \mathrm{v}$ ), elaboradas por el método de moldeado (casting). Se utilizó un diseño factorial $2^{3}$ con optimización de múltiples respuestas. Las concentraciones óptimas fueron pectina $(5,0 \% \mathrm{p} / \mathrm{v})$, glicerol $(4,5 \% \mathrm{p} / \mathrm{v})$ y aceite $(4,0$ $\%$ p/v) Se obtuvo elongación del 33,12 \pm 4,39 \%, tracción de 18,09 $\pm 2,98 \mathrm{MPa}$, esfuerzo de ruptura de 7,13 $\pm 0,60 \mathrm{~N}$ y módulo de Young de 105,04 $\pm 13,94 \mathrm{MPa}$. El glicerol proporcionó elongación, mientras que la pectina y el aceite mejoran la resistencia a la tracción. El material se ha desarrollado pensando en su posible aplicación como revestimiento de fibras naturales ya que este material puede mejorar la estabilidad de la fibra y sus propiedades mecánicas.

\section{Mechanical Properties of a Pectin Material for Coating Natural Fibers used in Agricultural Applications}

\begin{abstract}
The objective of this study was to evaluate the mechanical properties: percentage of elongation (\%) and tensile strength (MPa) of pectin films ( $4 \%$ and $6 \% \mathrm{w} / \mathrm{v})$, mineral oil $(2 \%$ and $6 \% \mathrm{p} / \mathrm{v})$ and glycerol $(3 \%$ and $6 \% \mathrm{w} / \mathrm{v})$, prepared by the casting method. A factorial design $2^{3}$ with optimization of multiple responses was used. The optimal concentrations were: pectin $(5.0 \% \mathrm{w} / \mathrm{v})$, glycerol $(4.5 \% \mathrm{w} / \mathrm{v})$ and oil $(4.0 \% \mathrm{w} / \mathrm{v})$, was obtained elongation $33.12 \pm 4.39 \%$, tensile strength $18.09 \pm 2.98 \mathrm{MPa}$, rupture effort of $7.13 \pm 0.60 \mathrm{~N}$ and Young's modulus of $105.04 \pm 13.94 \mathrm{MPa}$. The glycerol provided elongation, while the pectin and oil improve the tensile strength. The material has been developed thinking about its possible application as a coating of natural fibers, since this new material can improve the stability of the fiber and its mechanical properties.
\end{abstract}

Keywords: biodegradable material; pectin; elongation; tensile strength; natural fiber 


\section{INTRODUCCIÓN}

El cultivo de Arveja es gran importancia en el departamento de Nariño (Colombia)(Osorio et al., 2016), siendo es el primer productor de arveja (Pisum sativum) con 12.110 Ha sembradas y una producción de 27.266 ton para el año 2014 (AGRONET, 2015), para tutorar el cultivo se necesitan aproximadamente $9 \mathrm{Km}$. Ha ${ }^{-1}$ de fibra de polipropileno (Checa, 2015), este material al ser desechado causa graves problemas de contaminación en el suelo y puede afectar al ganado que se alimenta cerca al cultivo, se ha buscado reemplazar la fibra de polipropileno por fibras naturales como el algodón y el fique. Las fibras vegetales en los últimos años han adquirido una importancia no solo por su uso artesanal sino también industrial (Tinjacá et al., 2012). Las fibras naturales son baratas, son ligeras, tienen propiedades adecuadas, facilidad de separación, posibilidad de recuperación de la energía, neutralización al $\mathrm{CO}_{2}$, biodegradabilidad, reciclables y por eso resultan interesantes (Majewski Y Bledzki, 2013).

Por otra parte, los plásticos sintéticos como el polipropileno son económicos, livianos, resistentes a la oxidación, inalterables a los agentes atmosféricos y versátiles, sin embargo, estas mismas ventajas pueden ser sus peores inconvenientes. La alta resistencia a la corrosión, al agua y a la descomposición bacteriana los convierte en residuos difíciles de eliminar y, consecuentemente, en un grave problema ambiental (Valero et al., 2013) El polipropileno pueden tardar hasta 500 años en descomponerse (Gross y Kalra, 2002). Los plásticos sintéticos han contribuido a la contaminación ambiental provocada por desechos sólidos de baja degradabilidad, esto ha impulsado la búsqueda de sustitutos biodegradables (Acosta et al., 2006). El reemplazo total de los plásticos sintéticos por materiales biodegradables no se ha logrado hasta el presente, no obstante si se han sustituido algunos polímeros sintéticos por otros naturales, en aplicaciones específicas. Los polímeros sintéticos están siendo reemplazados gradualmente por materiales biodegradables de fuentes naturales en algunas aplicaciones a nivel agrícola e industrial (Villada et al., 2007). Rubio (2012) resalta que los materiales biodegradables se utilizan actualmente en diversos sectores, teniendo en cuenta las características funcionales que debe presentar el material según la aplicación específica a la que se destine. Los materiales elaborados con biopolímeros puede mejorar las propiedades de biodegradabilidad de materiales convencionales y mejorar sus propiedades mecánicas, Cruz et al. (2013) manifiestan que estas dependen del tipo de polímero y de los aditivos que sean usados para su elaboración.

Entre los polímeros naturales que se han utilizado se destacan los polisacáridos como la pectina. Las pectinas son polisacáridos presentes en los tejidos vegetales, compuestos principalmente por cadenas de ácido galacturónico. Las pectinas se han extraído por diferentes métodos de los tejidos vegetales de diversos frutos, principalmente de los materiales de desecho como por ejemplo de la pomaza de manzana y de las cáscaras de cítricos, en los cuales se ha encontrado un mayor rendimiento (Canteri et al., 2012). Recientemente se han encontrado reportes del empleo de pectinas para la fabricación de recubrimientos y películas de empaque como alternativa a los empaques de origen sintético, con lo cual pueden aprovecharse los desechos o subproductos de la producción agrícola (Aldana et al., 2011, Pérez et al., 2014), sin embargo no se ha reportado el uso de materiales biodegradables como revestimiento de fibras naturales por lo cual el objetivo de este estudio es el desarrollo de un material a base de pectina para el revestimiento de fibras naturales que mejoren sus propiedades mecánicas y sustituyan las fibras de polipropileno en el tutorado del cultivo de arveja ( $P$ sativum).

\section{METODOLOGÍA.}

La presente investigación se realizó en el laboratorio de investigación en conservación y calidad de alimentos de la universidad de Nariño - sede torobajo a 2527 MSNM.

\section{Materiales}

Para la elaboración del material se utilizó pectina cítrica de alto metoxilo, Glicerol USP, Aceite mineral, Tween 80 y Ácido cítrico (30\% p/v). Productos adquiridos en Químicos la Merced S.A.S - Pasto.

\section{Elaboración de películas}

La elaboración del material se realizó por el método Casting o moldeo en placa que consistió en realizar una disolución de pectina en agua destilada a $60^{\circ} \mathrm{C}$ previamente acidificada con una solución al $30 \%$ p/v de ácido cítrico hasta alcanzar pH 3,0; para evitar grumos se colocó en un agitador mecánico (IKA RW20 digital) al $1.200 \mathrm{rpm}$ por 5 minutos; por aparte se disolvieron el glicerol, el aceite mineral y el tween 80 , se agregaron a la solución de pectina y se agito a $1.500 \mathrm{rpm}$ por 5 minutos. En cajas de Petri $(135 \mathrm{~mm} \times 50 \mathrm{~mm})$ se vertieron $30 \mathrm{ml}$ de solución formadora y se llevaron a secado en incubadora de aire forzado (Gemmyco IN-601) a $45^{\circ} \mathrm{C}$ por 24 horas. Se retiraron las películas del molde y se cortaron en láminas de $2 \mathrm{~cm} \times 9 \mathrm{~cm}$. 


\section{Evaluación mecánica}

Las propiedades mecánicas de las películas se evaluaron con base a la norma técnica internacional ASTM D882-3 en un Texturometro Lloyd LS1 con mordazas para evaluación de películas y celda de carga de $1 \mathrm{KN}$.

\section{Diseño experimental}

Se utilizó un diseño de experimentos multifactorial $2^{3}$, se utilizaron como factores de estudio la concentración de pectina $(4 \%$ y $6 \%$ p/v) aceite mineral $(2 \%$ y $6 \%$ p/v) y glicerol como plastificante $(3 \%$ y $6 \% \mathrm{p} / \mathrm{v})$ para un total de 8 tratamientos (Tabla 1), se realizaron 5 réplicas para cada tratamiento para un total de 40 corridas experimentales, como variables de respuesta se evaluaron el porcentaje de elongación (\%) y resistencia a la tracción (MPa). El diseño se analizó mediante un análisis de varianza ANOVA al 95\% de confiabilidad y se determinó la mejor formulación con un análisis de optimización de múltiples respuestas. Se utilizó el software Statgraphics Centurión XV.II.

Tabla 1: Diseño de experimentos multifactorial $2^{3}$ para la elaboración de un material elaborado con pectina.

\begin{tabular}{|c|c|c|c|}
\hline Tratamiento & Pectina (\%) & Glicerol (\%) & Aceite (\%) \\
\hline T1 & 4 & 3 & 2 \\
\hline T2 & 4 & 3 & 6 \\
\hline T3 & 4 & 6 & 2 \\
\hline T4 & 4 & 6 & 6 \\
\hline T5 & 6 & 3 & 2 \\
\hline T6 & 6 & 3 & 6 \\
\hline T7 & 6 & 6 & 2 \\
\hline T8 & 6 & 6 & 6 \\
\hline
\end{tabular}

\section{Caracterización del material optimizado}

Se realizó una caracterización del material de revestimiento óptimo: Propiedades mecánicas, permeabilidad al vapor de agua, porcentaje de humedad: actividad de agua: espectroscopia de infrarrojo por transformada de fourier análisis termogravimétrico y calorimetría diferencial de barrido:

Propiedades mecánicas: se evaluaron con base a la norma técnica internacional ASTM D882-3 en un Texturometro Lloyd LS1 con mordazas para evaluación de películas y celda de carga de $1 \mathrm{KN}$. Las propiedades mecánicas de evaluación fueron: porcentaje de elongación (\%), resistencia a la tracción ( $\mathrm{MPa})$, esfuerzo máximo de ruptura (N) y módulo de Young (MPa).

Permeabilidad al vapor de agua: El material se acondicionó por 24 horas a una humedad relativa de 10-15\% y temperatura $17 \pm 2^{\circ} \mathrm{C}$ en un desecador de vidrio utilizando un $1 \mathrm{~kg}$ de potasa caustica $(\mathrm{KOH})$. Se colocaron las películas en la base de recipientes de plástico o pesafiltros sin tapa de dimensiones específicas y en su interior $3 \mathrm{~g}$ de sílica gel, luego se ajustó la película a la base con bandas de caucho y se pesó $\left(\mathrm{P}_{0}\right)$. Se colocaron en un desecador con agua destilada para generar un ambiente húmedo de aproximadamente 97$100 \%$ H.R. a temperatura ambiente $20 \pm 2{ }^{\circ} \mathrm{C}$. Se registraron los peso del envase con las películas $\left(P_{t}\right)$ cada: $1,2,4,6,8,12,16,24,32$ horas. La permeabilidad al vapor de agua se calculó la permeabilidad al vapor de agua utilizando la ecuación 1.

$$
P V A=\frac{M p t}{A} * \frac{e}{\Delta P v}
$$

Donde PVA es la permeabilidad al vapor de agua $\left(\mathrm{g} \cdot \mathrm{s}^{-1} \cdot \mathrm{m}^{-1} \cdot \mathrm{Pa}^{-1}\right), M p t$ es la velocidad de transferencia de vapor de agua $\left(\mathrm{g} \cdot \mathrm{s}^{-1}\right)$ y que equivale a la pendiente de la recta entre la ganancia de peso en el tiempo, A es el área de contacto de la película con el medio $\left(\mathrm{m}^{2}\right)$, e es espesor de la película $(\mathrm{m}) \Delta P v$ es la diferencia de presión de vapor de la atmosfera del desecador y la atmosfera de la sílica gel a las condiciones de temperatura y humedad relativas que se encuentre el montaje (3170 Pa).

Porcentaje de humedad: se utilizó una balanza de humedad KERN DBS, la evaluación se hizo a $105^{\circ} \mathrm{C}$ con un porcentaje de variabilidad de $0,05 \%$. Actividad de agua: se utilizó un medidor de humedad Rotronic, la evaluación se hizo a temperatura ambiente.

Espectroscopia de infrarrojo por transformada de Fourier: los análisis se realizaron en un equipo IR TRACER100 marca Shimadzu. Se realizaron los análisis tanto para pectina como para el material optimizado, Las 
muestras se secaron a $60^{\circ} \mathrm{C}$ y se leyeron directamente en MID-DRIFTs $\left(400-4000 \mathrm{~cm}^{-1}\right.$ - Diffuse Reflectance Infrared Fourier Transform Spectroscopy), con la adición de KBr para el material.

Análisis termogravimétrico y calorimetría diferencial de barrido: los análisis se realizaron en un equipo SDTQ600, $2 \mathrm{mg}$ de muestra se someten a atmósfera de nitrógeno, a una velocidad de flujo de $100 \mathrm{ml} / \mathrm{min}$, de 25 a $800 \stackrel{\circ}{\circ}$ con una velocidad de calentamiento de $10 \stackrel{\circ}{\circ} / \mathrm{min}$.

\section{RESULTADOS Y DISCUSIÓN}

Se obtuvieron películas del material de pectina con un espesor de 0,14 $\pm 0,03 \mathrm{~mm}$ y una humedad de $9,13 \pm$ $0,61 \%$. Los resultados de la evaluación mecánica del material elaborado con pectina se presentan en la tabla 2 (Letras diferentes indican diferencias significativas entre los tratamientos). La evaluación del diseño de experimentos indico el efecto significativo de cada uno de los factores y sus interacciones sobre las propiedades mecánicas (Figuras 1 y 2).

Tabla 2: Propiedades mecánicas, porcentaje de elongación y resistencia a la tracción del material elaborado con pectina.

\begin{tabular}{|c|c|c|}
\hline Tratamiento & Porcentaje de elongación (\%) & Resistencia a la tracción (MPa) \\
\hline T1 & $12,603 \pm 1,19^{\mathrm{b}}$ & $10,37 \pm 2,17^{\mathrm{d}}$ \\
\hline T2 & $12,330 \pm 0,83^{\mathrm{b}}$ & $7,04 \pm 0,96^{\mathrm{c}}$ \\
\hline T3 & $38,077 \pm 4,18^{\mathrm{f}}$ & $6,09 \pm 1,47^{\mathrm{bc}}$ \\
\hline T4 & $29,353 \pm 2,18^{\mathrm{e}}$ & $3,08 \pm 0,38^{\mathrm{a}}$ \\
\hline T5 & $4,3152 \pm 0,86^{\mathrm{a}}$ & $15,79 \pm 2,36^{\mathrm{e}}$ \\
\hline T6 & $5,064 \pm 1,06^{\mathrm{a}}$ & $6,12 \pm 0,86^{\mathrm{bc}}$ \\
\hline T7 & $15,952 \pm 1,21^{\mathrm{c}}$ & $7,32 \pm 0,56^{\mathrm{c}}$ \\
\hline T8 & $18,723 \pm 0,96^{\mathrm{d}}$ & $5,25 \pm 0,49^{\mathrm{b}}$ \\
\hline
\end{tabular}

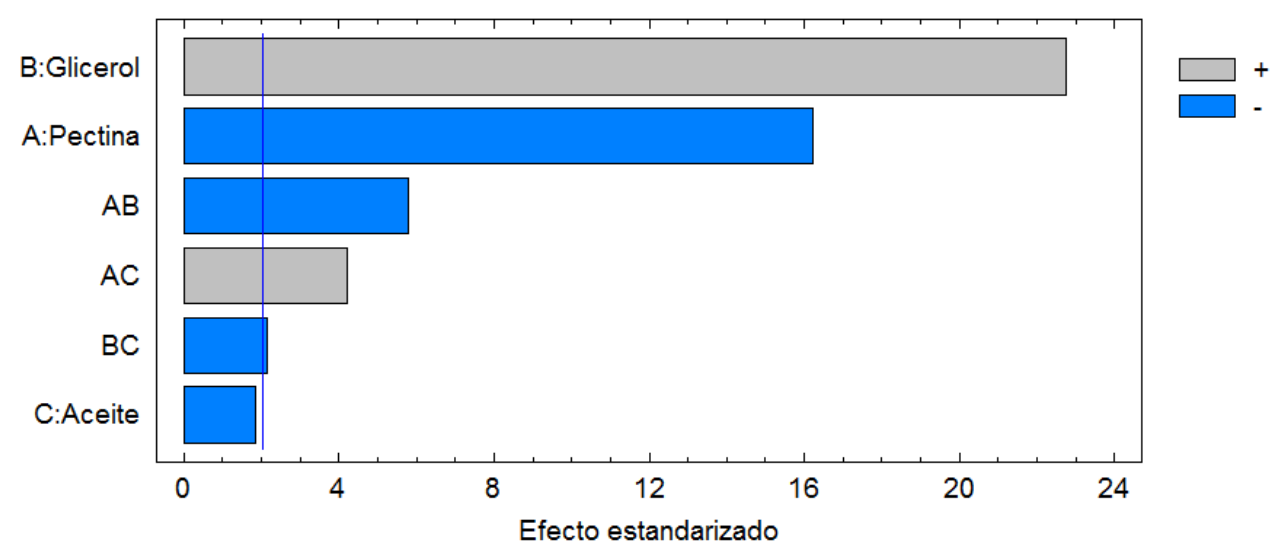

Fig. 1: Diagrama de Pareto estandarizado para el porcentaje de elongación (\%) del material elaborado con pectina.

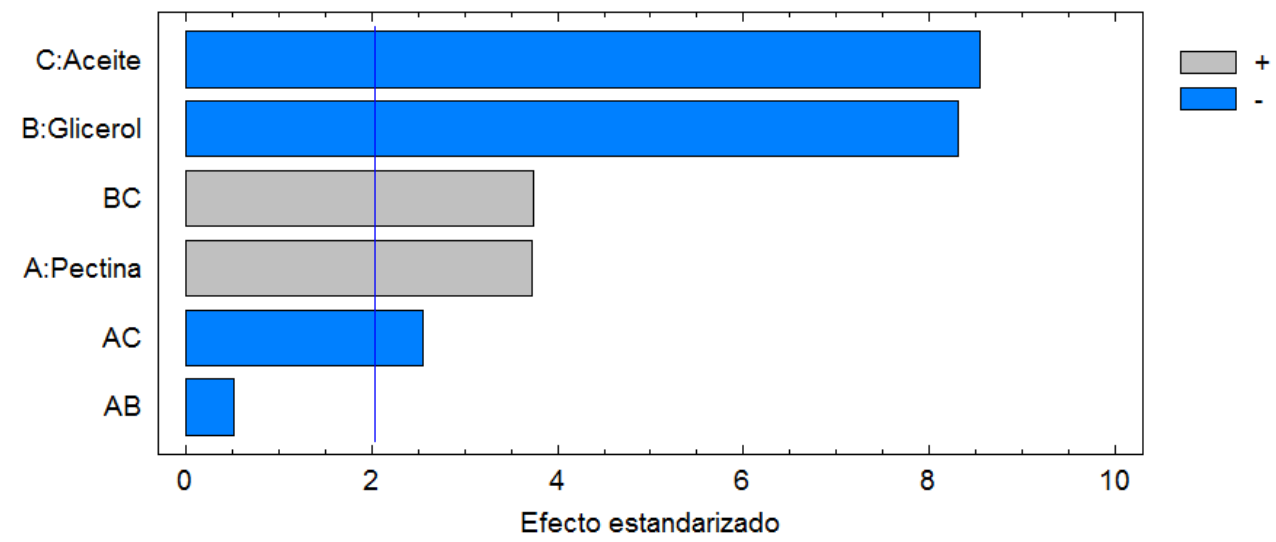

Fig. 2: Diagrama de Pareto estandarizado para la resistencia a la tracción (MPa) del material elaborado con pectina. 


\section{Evaluación del porcentaje de elongación}

El análisis de varianza indica que el glicerol tiene un efecto significativo positivo sobre la elongación ( $p$-valor $=0,0000)$, de igual manera la interacción de pectina-aceite ( $p$-valor $=0,0002)$, la pectina tiene un efecto significativo negativo ( $p$-valor $=0,0000)$, al igual que las interacciones pectina-glicerol $(p$-valor $=0,0000)$ y glicerol-aceite ( $p$-valor $=0,0382$ ), el aceite por sí solo no tiene influencia sobre la elongación (Figura 1). El modelo matemático que representa el comportamiento de la elongación del material es: Elongación =1,76214 $-2,71703^{\star}$ Pectina $+13,8883^{*}$ Glicerol $-3,04797^{*}$ Aceite $-1,43345^{\star}$ Pectina*Glicerol $+0,782232^{\star}$ Pectina ${ }^{\star}$ Aceite - 0,267893*Glicerol*Aceite. El modelo tiene un $\mathrm{R}^{2}$ (ajustado) de $95,53 \%$.

Es válido afirmar que la concentración de glicerol es la que proporciona elongación al material, el glicerol funciona como plastificante en la composición brindando mayor movilidad a las moléculas de pectina. Al incrementar la concentración de plastificante en los rangos estudiados podemos evidenciar una mayor elongación, sin embargo la concentración de pectina influye negativamente en la variable porcentaje de elongación por esta razón los tratamientos que contenían altas concentraciones de pectina no lograron la elongación que obtuvieron los tratamientos con menor concentración de este componente; por otra parte el aceite mineral no tiene un efecto significativo sobre la elongación.

La adición de plastificantes como glicerol modifica la estructura de la red formada por el biopolímero, logrando películas con una alta elasticidad (Moncayo, 2013). Sánchez et al. (2014) indica que con el incremento en la concentración de plastificante reduce las fuerzas intermoleculares con lo que se aumenta la flexibilidad y se reduce la resistencia de los materiales. Lozano et al. (2016) Indican que para aumentar la capacidad de estiramiento de las películas, el glicerol actúa químicamente entre las cadenas poliméricas moleculares, reduciendo las fuerzas de cohesión y permitiendo el estiramiento de la estructura, en su estudio, la capacidad de estiramiento disminuyo con la explicación de extractos pécticos; este mismo efecto se presenta en el presente estudio donde el aumento en la concentración de pectina disminuyo la elongación de las películas.

\section{Evaluación de la resistencia a la tracción (MPa).}

El análisis de varianza indica que el aceite y el glicerol tienen un efecto significativo negativo sobre la tracción ( $p$-valor $=0,0000)$, de igual manera la interacción de pectina-aceite $(p$-valor $=0,0155)$, por otra parte la pectina y la interacción glicerol-aceite tienen un efecto significativo positivo ( $p$-valor $=0,0007$ ), la interacción pectinaglicerol no tiene efecto significativo sobre la resistencia a la tracción (Figura 2). El modelo matemático que representa el comportamiento de la resistencia a la tracción del material es: Tracción=10,9159+ $2,75291^{\star}$ Pectina- $2,32088^{\star}$ Glicerol $-0,926961^{\star}$ Aceite $-0,0926774^{\star} P$ ectina ${ }^{\star}$ Glicerol $-0,337575^{\star} P e c t i n{ }^{*}$ Aceite $+0,33005^{\star}$ Glicerol ${ }^{\star}$ Aceite. El modelo tiene un $\mathrm{R}^{2}$ (ajustado) de $81,40 \%$.

La resistencia a la tracción funciona de manera inversa a la elongación, laminas con mayor elongación tienen menor resistencia de tracción por eso los componentes actúan de manera inversa, la pectina mejora las características de resistencia de la película. El aceite seguramente por ser un aditivo líquido proporciona flexibilidad y no rigidez. La resistencia a la tracción explica la resistencia mecánica de la película debido a las fuerzas de cohesión entre cadenas, mientras que el alargamiento a la rotura mide su plasticidad, que es la capacidad de una película de extenderse antes de romperse (Galus y Lenart, 2013). Debido a la naturaleza estructural de esos atributos, usualmente películas con alta resistencia a la tracción muestran un bajo alargamiento a la rotura de modo que ambas propiedades deben ser analizadas simultáneamente (Altenhofen et al., 2009). La resistencia a la tracción es la propiedad mecánica de mayor interés en el material porque se busca reforzar fibras naturales con un revestimiento capaz de mantener su estructura a pesar de la tensión que se pueda ejercer sobre la fibra, sin dejar de lado la elongación necesaria para no tornarse en un material quebradizo.

\section{Optimización de múltiples respuestas.}

La optimización de múltiples respuestas se realizó teniendo en cuenta que se deseaba obtener un material con mayor resistencia que elongación por lo tanto se asignó un impacto a la variable de elongación (\%) del $30 \%$ y resistencia a la tracción ( $\mathrm{MPa}$ ) un impacto de $70 \%$. Los resultados indicaron que las concentraciones óptimas son: pectina $(5,0 \% \mathrm{p} / \mathrm{v})$, glicerol $(4,5 \% \mathrm{p} / \mathrm{v})$ y aceite $(4,0 \% \mathrm{p} / \mathrm{v})$. La figura 3 indica la superficie de respuesta para la optimización de múltiples variables.

\section{Caracterización del material optimizado.}

A continuación se presentan las propiedades mecánicas, permeabilidad al vapor de agua, contenido de humedad, actividad de agua, análisis de espectroscopia de infrarrojo, análisis termogravimétrico y calorimetría diferencial de barrido del material obtenido. 


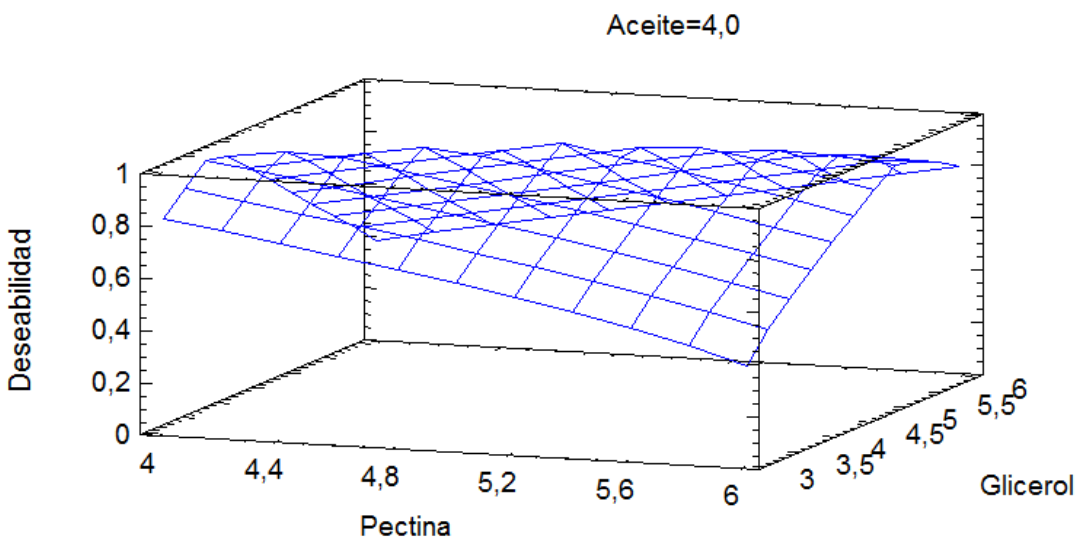

Fig. 3: Superficie de respuesta estimada para la optimización de múltiples respuestas entre elongación (\%) y resistencia a la tracción (MPa) del material.

\section{Propiedades mecánicas.}

La evaluación mecánica del material elaborado con la formulación optimizada indicó un porcentaje de elongación del $33,12 \pm 4,39 \%$, resistencia a la tracción de $18,09 \pm 2,98 \mathrm{MPa}$, esfuerzo de ruptura de 7,13 \pm $0,60 \mathrm{~N}$ y módulo de Young de 105,04 $\pm 13,94 \mathrm{MPa}$. Se han realizado algunos estudios sobre el comportamiento mecánico de películas de pectina con inclusión de otros biopolímeros; peliculas con pectina y alginato a diferentes concentraciones de calcio presentaron una elongación de 2,1\% a 19,8\% (bajas concentraciones de calcio) y del 1,5\% al $12,9 \%$ para concentraciones altas de calcio, no solo el calcio mejor la elongación sino también la incorporación de alginato. Películas de pectina con metilcelulosa y glucamano en proporciones de 1:1:4 respectivamente tuvieron un porcentaje de elongación 9,85\% y una resistencia a la tracción de 72,63 MPa (Chambi y Grosso, 2011).

Películas de pectina de bajo metoxilo tuvieron porcentaje de elongación de 5,9 $\pm 1,2 \%$ y resistencia a la tracción de 22,4 $\pm 2,4 \mathrm{MPa}$ (Galus y Lenart, 2013). Pasini et al. (2016) indico que el incremento de concentración de glicerol aumenta la elongación obteniendo valores de $4,83 \%$ a $71,64 \%$ pero se reduce la resistencia a la tracción de 69,33 a 4,48 MPa, además se optime una reducción del módulo de Young de 3270 a 6,1 MPa. Vinicius et al. (2016) realizaron películas a base de pectina de alto metoxilo que tuvieron capacidad de estiramiento de $1,79 \%$ y resistencia a la tracción de 30,81 MPa y con la incorporación de quitosano lograron incrementarla a 2,22 \% el estiramiento y a $58,51 \mathrm{MPa}$ la resistencia a la tracción. Los resultados que presentan varios autores son variados porque dependen de la concentración, espesor y contenido de humedad sin embargo los datos obtenidos en este estudio son similares.

\section{Permeabilidad al vapor de agua.}

El material optimizado tuvo un una permeabilidad al vapor de agua (PVA) de 5,975 $\times 10^{-10} \mathrm{~g} \cdot \mathrm{s}^{-1} \cdot \mathrm{m}^{-1} \cdot \mathrm{Pa}^{-1}$. La PVA es importante porque indica la cantidad de agua que puede atravesar el material, se espera que la permeabilidad tenga valores muy pequeños, los valores obtenidos indican que el material tiene un grado de resistencia importante al vapor de agua y puede mantenerse viable por algún tiempo en condiciones ambientales de baja humedad. Algunos estudios sobre permeabilidad al vapor de agua reportan similares resultados a los obtenidos. Películas de carragenano y pectina tuvieron una PVA entre $1,4 \times 10^{-11}$ a $4,4 \times 10^{-}$ ${ }^{11} \mathrm{~g} \cdot \mathrm{s}^{-1} \cdot \mathrm{m}^{-1} \cdot \mathrm{Pa}^{-1}$ (Alves et al., 2010). Galus y Lenart (2013) obtuvieron una PVA entre $0,84 \times 10^{-10}$ y $1,73 \times 10^{-}$ $10 \mathrm{~g} \cdot \mathrm{s}^{-1} \cdot \mathrm{m}^{-1} \cdot \mathrm{Pa}^{-1}$ para películas de bajo metoxilo. Películas con extractos pécticos de limón tuvieron una PVA de $5,17 \times 10^{-11} \mathrm{~g} \cdot \mathrm{s}^{-1} \cdot \mathrm{m}^{-1} \cdot \mathrm{Pa}^{-1}$ (Sánchez et al., 2014). Películas de pectina de frutos de tejocote y cera de candelilla obtuvieron PVA entre $9,3 \times 10^{-14}$ y $1,3 \times 10^{-14} \mathrm{~g} \cdot \mathrm{s}^{-1} \cdot \mathrm{m}^{-1} \cdot \mathrm{Pa}^{-1}$ (Lozano et al., 2016). Pasini et al. (2016) reporta una PVA de $1,5 \times 10^{-9} \mathrm{~g} \cdot \mathrm{s}^{-1} \cdot \mathrm{m}^{-1} \cdot \mathrm{Pa}^{-1}$ en películas de pectina.

\section{Contenido de humedad y actividad de agua.}

El porcentaje de humedad del 6,79 $\pm 0,96 \%$, actividad de agua (AW) de 0,32 $\pm 0,02$. Es importante reconocer que la permeabilidad está ligada al contenido de humedad y actividad de agua del material, así mismo estas propiedades juegan un papel importante en la degradación, estabilidad y propiedades mecánicas del material. Eghbala et al. (2016) indica que el contenido de agua afectara fuertemente las propiedad mecánicas y de barrera. El agua también funciona como un plastificante y puede influir en la estructura y organización molecular.

Eghbala et al. (2016) obtuvo $14,5 \%$ de humedad aproximadamente en películas de caseinato de sodio y pectina de bajo metoxilo cuyas propiedades mecánicas fueron módulo de Young de 182,97 $\pm 6,48 \mathrm{MPa}$, 
resistencia a la tracción de 15,64 $\pm 1,74 \mathrm{MPa}$ y elongación a la rotura de 9,35 \pm 0,10\%. Duran et al., (2017) reporta un contenido de humedad entre $17,3 \%$ a $13,6 \%$ en películas quitosano y pectina donde variaron la elongación de 35,5 a 26\%, resistencia a la tracción de 25,9 a $31 \mathrm{MPa}$ y la PVA de 1,06 x 10-15 a 0,97 x 10-15 $\mathrm{g} \cdot \mathrm{s}^{-1} \cdot \mathrm{m}^{-1} \cdot \mathrm{Pa}^{-1}$

\section{Espectroscopia de infrarrojo por transformada de Fourier}

Los resultados de la espectroscopia de infrarrojo se indican en la figura 4. Los espectros infrarrojos indican la presencia de algunos grupos químicos funcionales; en la pectina se indica un pico a $3410 \mathrm{~cm}^{-1}$ posiblemente de grupos hidroxilo de moléculas de agua, picos entre 2370 y $2929 \mathrm{~cm}^{-1}$ posiblemente de grupos metilo característicos del ácido galacturónico, picos a frecuencia de $1747 \mathrm{~cm}^{-1}$ y $1631 \mathrm{~cm}^{-1}$ corresponde a grupos carboxilo esterificados y sin esterificación respectivamente, además picos a $1452 \mathrm{~cm}^{-1}$ indican grupos metoxilo, a $1236 \mathrm{~cm}^{-1}$ indican la presencia de grupos carbonilo. Los grupos característicos de sacáridos se evidencian entre los 1155 a $1006 \mathrm{~cm}^{-1}$, en los rangos de 700 a $900 \mathrm{~cm}^{-1}$ se evidencia la presencia de anillos benzoicos, posiblemente de la estructura de cadena (Figura 4). Los resultados obtenidos son similares a los reportados para caracterización de pectina (Vinicius et al., 2011; Shu-Ying et al., 2018).

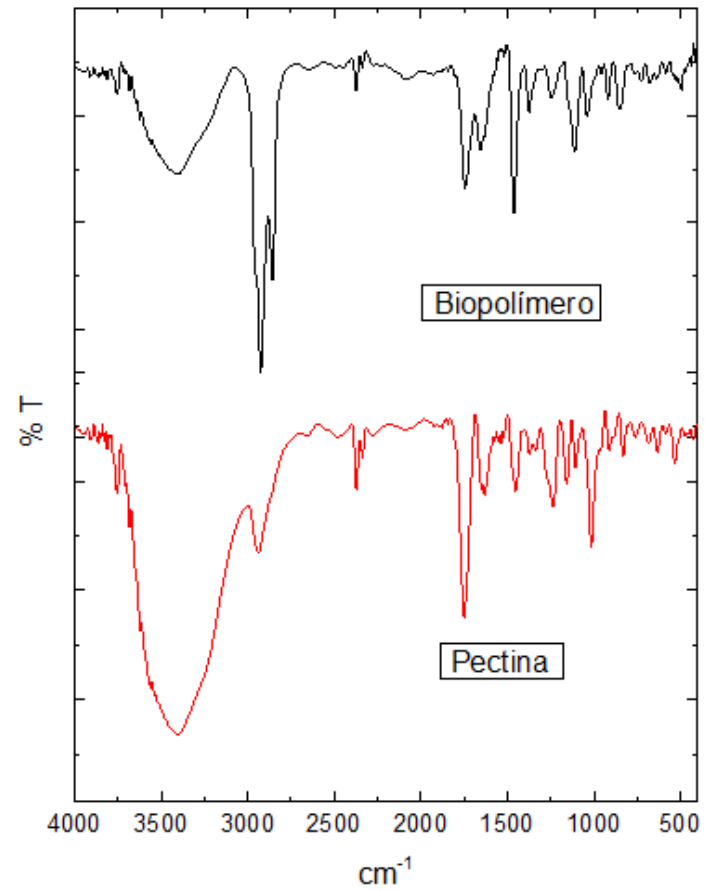

Fig. 4: Espectroscopia de infrarrojo por transformada de Fourier (FTIR) para material de revestimiento optimizado y pectina de alto metoxilo.

Los espectros infrarrojos del material indican la presencia de grupos hidroxilo a $3400 \mathrm{~cm}^{-1}$, grupos metilo del a 2929 y $2854 \mathrm{~cm}^{-1}$ con mayor estiramiento que en la pectina, posiblemente por la ruptura de las cadenas de ácido galacturónico, grupos carboxilo esterificados y sin esterificar a 1739 y $1631 \mathrm{~cm}^{-1}$ con menor estiramiento que en la pectina, sin embargo los grupos metoxilo identificados a $1462 \mathrm{~cm}^{-1}$ con mayor estiramiento. Los grupos indicadores de glicerol en el material pueden ubicarse en los $1006 \mathrm{~cm}^{-1}$ (Figura 4). Los resultados obtenidos son comparables a los reportados por algunos autores en la elaboración de materiales a base de pectina (Sánchez et al., 2014; Nesic et al., 2017).

\section{Análisis termogravimétrico y calorimetría diferencial de barrido}

El termograma para el material de revestimiento optimizado indica la pérdida de peso y el flujo de calor con el incremento de la temperatura (Figura 5). Se puede observar una pérdida de peso del $1,11 \%$ hasta los 105 ${ }^{\circ} \mathrm{C}$, posiblemente del agua contenida en el material, a los $275^{\circ} \mathrm{C}$ se presenta una pérdida de peso del $50,69 \%$ posiblemente correspondiente a la degradación de la pectina, componente mayoritario en el material. Se reportan perdidas de peso del $48 \%$ a una temperatura de $242,66^{\circ} \mathrm{C}$ para muestras con extractos de pectina (Sánchez et al., 2014), pérdidas del 50\% por encima de los $250^{\circ} \mathrm{C}$ (Nesic et al., 2017) y pérdidas del $60 \%$ en un rango de 180 a $285^{\circ} \mathrm{C}$ para muestras de pectina (Seslija et al., 2018). 


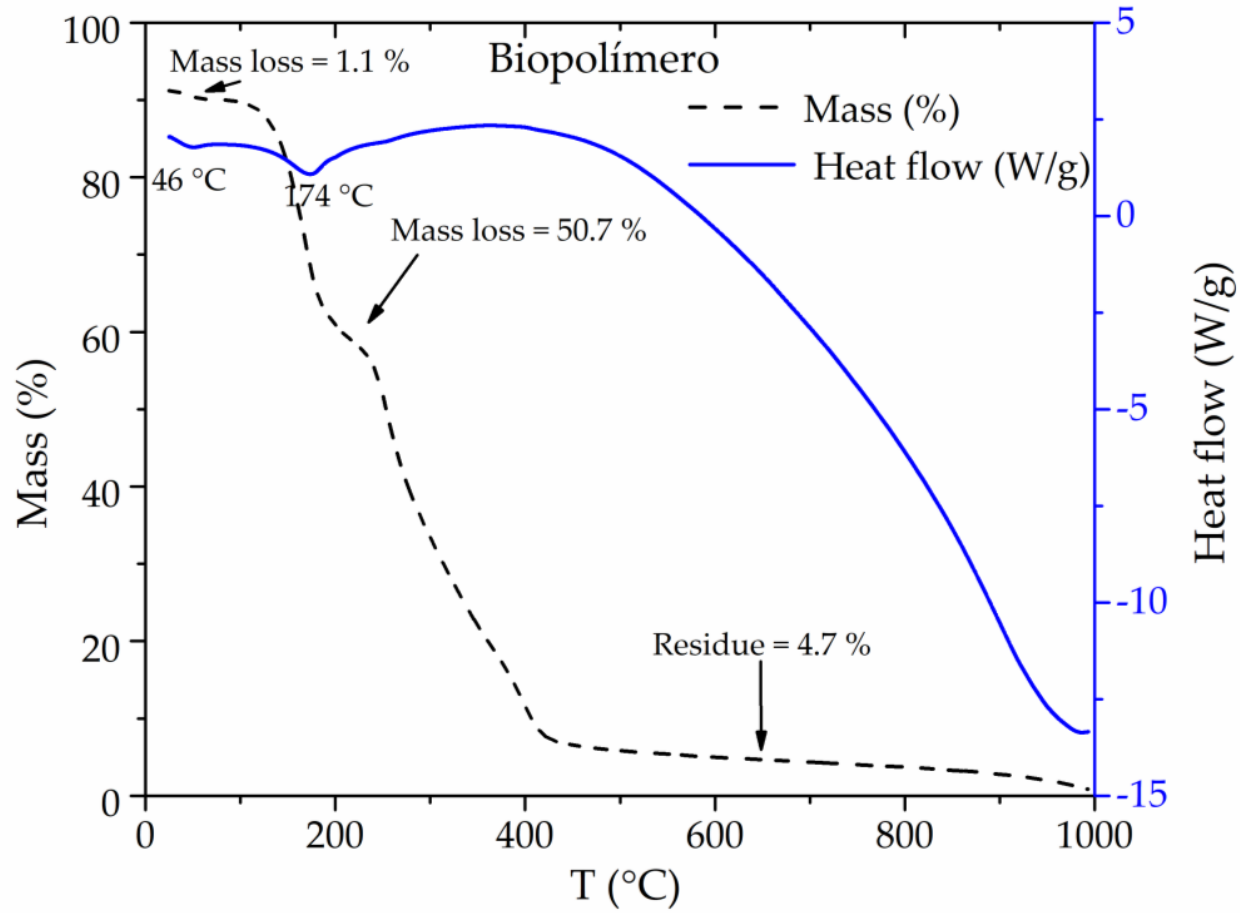

Fig. 5: Termogravimetria y calorimetría diferencial de barrido (TGA/DSC) para material de revestimiento optimizado y pectina de alto metoxilo.

Los resultados obtenidos en el análisis TGA/DSC indican el comportamiento térmico del material, sin embargo no es posible determinar claramente una temperatura de cristalización o fusión, a la temperatura de $174,93^{\circ} \mathrm{C}$ se presenta una leve disminución del flujo de calor que coincide con una relativa estabilidad en la pérdida de peso, mas no podemos asumir que se trate de una cristalización de la pectina. Nesic et al. (2017) reporta que en películas de pectina y glicerol la temperatura a la que inicia la degradación de la pectina es $195^{\circ} \mathrm{C}$ y el glicerol disminuye la temperatura debido a la generación de enlaces intermoleculares que genera una estructura más frágil de la pectina. Se reportan temperaturas de transición vítrea para la pectina de $131^{\circ} \mathrm{C}$ (Seslija et al., 2018).

\section{CONCLUSIONES}

El recubrimiento desarrollado tiene propiedades mecánicas adecuadas para mejorar la calidad de las fibras, propiedades de permeabilidad adecuadas y características químicas compatibles con el material a revestir. El material se ha desarrollado pensando en su posible aplicación como revestimiento de fibras naturales para mejorar sus propiedades mecánicas manteniendo estable la estructura de las fibras y garantizando sus características en un tiempo más prolongado. El recubrimiento de fibras naturales con biopolímeros como la pectina puede ser una alternativa para la sustitución de fibras de polipropileno en aplicaciones agrícolas como el tutorado de arveja (Pisum sativum) sin embargo es necesario evaluar el comportamiento mecánico y estructural en condiciones ambientales.

\section{AGRADECIMIENTOS}

Agradecimientos al sistema general de regalías - SGR (Colombia) por la financiación del proyecto de "Investigación para el mejoramiento de la tecnología de producción de arveja (Pisum sativum L.) en el departamento de Nariño" proyecto - Cod. BPIN 2013000100278.

\section{REFERENCIAS}

Acosta, H.A., H.S. Villada, G.A. Torres y J.G. Ramírez, Morfología superficial de almidones termoplásticos agrio de yuca y nativo de papa por microscopía óptica y de fuerza atómica, Información Tecnológica, 17(3), 63-70 (2006)

AGRONET, Red de Información y Comunicación del Sector Agropecuario Colombiano. Ministerio de Agricultura, Colombia Siembra, Evaluaciones agropecuarias municipales - Arveja (2015)

Aldana, D.S., C.N. Aguilar, J.C. Contreras y G.V. Nevárez, Moléculas pécticas: extracción y su potencial aplicación como empaque, Tecnociencia Chihuahua, 5(2), 76 - 82 (2011)

Altenhofen, M., A. Krause y T. Guenter, Alginate and pectin composite films crosslinked with $\mathrm{Ca}^{2+}$ ions: Effect of the plasticizer concentration, Carbohydrate Polymers, 77(4), 736 - 742 (2009) 
Alves, V.D., N. Costa y C. Isabe, Barrier properties of biodegradable composite films based on kappa-carrageenan/pectin blends and mica flakes, Carbohydrate Polymers, 79 (10), 269 - 276 (2010)

Canteri, A., L. Gilvan y A.P. Scheer, Pectina: de Materia Prima a Producto Final, Polímeros, 22(2), 149 - 157 (2012)

Chambi, H. y C. Grosso, Mechanical and water vapor permeability properties of biodegradables films based on methylcellulose, glucomannan, pectin and gelatin, Ciência e Tecnologia de Alimentos, 31(3), 739 - 746 (2011)

Checa, O., Mejoramiento de la tecnología de producción de arveja (Pisum sativum L.) en el departamento de Nariño, Consejo departamental de ciencia, tecnología e innovación CODECTI (2015)

Cruz, R., Y. Martínez y V. López, Biopolímeros y su integración con polímeros convencionales como alternativa de empaque de alimentos, Temas selectos de Ingeniería de Alimentos, 7(2), 42 - 52 (2013)

Duran, R., L.L, Pérez y otros tres autores, Production and characterization of films based on blends of chitosan from blue crab (Callinectes sapidus) waste and pectin from Orange (Citrus sinensis Osbeck) peel, International journal biological macromolecules, 98(79), 676-683 (2017)

Eghbala, N., M. Saeid y otros cuatro autores, Complex coacervation for the development of composite edible films based on LM pectin and sodium caseinate, Carbohydrate Polymers, 151, 947-956 (2016)

Galus, S. y A. Lenart, Development and characterization of composite edible films based on sodium alginate and pectin, Journal of Food Engineering, 115(4), 459 - 465 (2013)

Gross, R. y B. Kalra, Biodegradable polymers for the environment, Science, 297(5582), 803 - 807 (2002)

Lozano, M., S. Valley otros tres autores, Películas basadas en emulsiones de pectina de frutos de tejocote (Crataegus spp.) y cera de candelilla: caracterización y aplicación en Pleurotus ostreatus. Agrociencia, 50(7), 849 - 866 (2016)

Majewski, T. y A. Błędzki, Desarrollo y aplicaciones actuales de los plásticos reforzados por fibras naturales, Memorias del XIX congreso internacional anual de la somim (2013)

Moncayo, D., Desarrollo de un recubrimiento comestible a partir de un biopolímero para prolongar la vida útil de frutas frescas, Tesis de investigación: Magister en Ciencia y tecnología de Alimentos, Universidad Nacional de Colombia (2013)

Nesic, A., J. Ruiz y otros cuatro autores, Pectin-polyvinylpyrrolidone films: A sustainable approach to the development of biobased packaging materials, Composites Part B 110, 56 - 61 (2017)

Osorio, O., G. Rodríguez, F. Castellanos y A. Chávez, Procesamiento de Arvejas (Pisum sativum L.). Parte 3: cinética de perdida de agua en chips de Arveja en condiciones de frita convencional y a vacío, Información Tecnológica, 27(4), 33$42(2016)$

Pasini, S.D., E.A. Takara, J. Marchese y N.A. Ochoa, Influence of plasticizers in pectin films: Microstructural changes, Materials Chemistry and Physics, 162(15), 491 - 497 (2016)

Pérez, P.J., W.X. Du y otros tres autores, Edible films from pectin: Physical-mechanical and antimicrobial properties - A review, Food Hydrocolloids, 35(1), 287 - 296 (2014)

Rubio, M. y J.A. Guerrero, Polímeros utilizados para la elaboración de películas biodegradables, Temas selectos de Ingeniería de Alimentos, 6(2), 173 - 181 (2012)

Sánchez, D. J.C., Contreras, G.V. Nevárez y C.N. Aguilar, Caracterización de películas comestibles a base de extractos pécticos y aceite esencial de limón Mexicano. CyTA - Journal of Food, 13(1), 17- 25 (2014)

Seslija, S., S., Pavle y otros cinco autores, Physico-chemical evaluation of hydrophobically modified pectin derivatives: Step toward application, International Journal of Biological Macromolecules, https://doi.org/10.1016/j.ijbiomac.2018.03.006 (2018)

Shu-Ying. Xua., L. Jun-Peng y otros ocho autores, Ultrasonic-microwave assisted extraction, characterization and biological activity of pectin from jackfruit peel, LWT - Food Science and Technology, 90, 577 - 582 (2018)

Tinjacá, B.P., V. Slone y N. Arango, El fique: fibra natural que construye sociedad, Proyecto de investigación, Fundación Universitaria del área Andina (2012)

Valero, M.F., Y. Ortegón e Y. Uscategui, Biopolímeros: avances y perspectivas. Dyna, 80(131), 171 - 180 (2013)

Villada, H.S., H.A. Acosta. y R.J. Velasco, Biopolímeros naturales usados en empaques biodegradables, Temas Agrarios, 12(2), 5 - 13 (2007)

Vinicius, M., C. Gomide, R. Marcia y L. Capparelli, Chitosan nanoparticles on the improvement of thermal, barrier, and mechanical properties of high- and low-methyl pectin films. Food Hydrocolloids, 52(1), 732 - 740 (2011) 
